\title{
COVERS OF GENERALIZED QUADRANGLES
}

\author{
JOSEPH A. THAS AND KOEN THAS
}

ABSTRACT. We solve a problem posed by Cardinali and Sastry [2] about factorization of 2-covers of finite classical generalized quadrangles. To that end, we develop a general theory of cover factorization for generalized quadrangles, and in particular we study the isomorphism problem for such covers and associated geometries. As a byproduct, we obtain new results about semipartial geometries coming from $\theta$-covers, and consider related problems.

\section{CONTENTS}

1. Introduction 2

2. Definitions 3

3. Covers 4

4. Factorization of morphisms, I - lower decomposition 5

5. Factorization of morphisms, II — higher decomposition 8

6. The original question 9

7. More on subtended ovoids 10

8. The geometry $\Gamma\left(\mathcal{S}, \mathcal{S}^{\prime}\right)=\mathcal{E} \quad 11$

9. Uniqueness of covers 12

10. Particular case 13

11. Subtending in the Kantor-Knuth quadrangles $\quad 15$

$\begin{array}{lll}\text { Appendix A. Extension in nonclassical cases } & 17\end{array}$

References 19

2000 Mathematics Subject Classification. 05B25; 05E18; 51A10; 51B25; 51E12.

Key words and phrases. cover; generalized quadrangle; morphism; semipartial geometry; subtended ovoid. 


\section{INTRODUCTION}

In a recent paper [2], Cardinali and Sastry study various aspects of geometries related to ovoids of the classical finite generalized quadrangle $\mathcal{W}\left(2^{n}\right)$. In particular, starting with the natural embedding $\mathcal{W}\left(2^{n}\right) \hookrightarrow \mathcal{Q}\left(5,2^{n}\right)$, they consider the geometry $\mathcal{A}$ defined by $Q\left(5,2^{n}\right) \backslash \mathcal{W}\left(2^{n}\right)$, and the geometry $\mathcal{E}$ which consists of elliptic ovoids in $\mathcal{W}\left(2^{n}\right)$ as points, and rosettes of such ovoids as lines (more formal details can be found in $\S 2$ and $\S 3$ ). There is a natural projection $\pi: \mathcal{A} \mapsto \mathcal{E}$. They then ask if any 2-cover $\gamma: \mathcal{A} \mapsto \mathcal{E}$ factors through $\pi$ and an automorphism $\widetilde{\alpha}$ of $\mathcal{A}$ (or $Q\left(5,2^{n}\right)$ ):

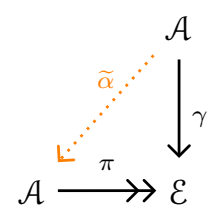

This is the starting point of the present paper, and our first main result is an answer of that question in the concrete case of Cardinali and Sastry - the answer is positive. Our setting is without finiteness restrictions, and without assuming that the quadrangles in question are classical. We just impose enough restrictions such that the geometries $\mathcal{A}$ and $\mathcal{E}$ exist. (The latter geometry will have as points subtended ovoids, and as lines rosettes of such ovoids.) More precisely, we have:

Theorem 1.1. Let $\mathcal{S}$ be a thick generalized quadrangle, and let $\mathcal{S}^{\prime} \hookrightarrow \mathcal{S}$ be a thick full subquadrangle which is a geometrical hyperplane of $\mathcal{S}$. Define $\mathcal{A}$ and $\mathcal{E}$ as above, and let $\pi: \mathcal{A} \mapsto \mathcal{E}$ be the natural projection. Then every cover $\gamma: \mathcal{A} \mapsto \mathcal{E}$ factorizes as $\gamma=\pi \circ \widetilde{\alpha}$, with $\widetilde{\alpha}$ an automorphism of $\mathcal{A}$, if and only if every automorphism of $\mathcal{E}$ is induced by an automorphism of $\mathcal{S}$.

In fact, we also obtain a decomposition property at the level of the geometry $\mathcal{E}$, which is used for proving the latter theorem:

Theorem 1.2. Let $\mathcal{S}$ be a thick generalized quadrangle, and let $\mathcal{S}^{\prime} \hookrightarrow \mathcal{S}$ be a thick full subquadrangle which is a geometrical hyperplane of $\mathcal{S}$. Define $\mathcal{A}$ and $\mathcal{E}$ as above, and let $\pi: \mathcal{A} \mapsto \mathcal{E}$ be the natural projection. Then any cover $\gamma: \mathcal{A} \mapsto \mathcal{E}$ factorizes as $\gamma=\alpha \circ \pi$, with $\alpha$ a unique automorphism of $\mathcal{E}$.

In categorical terms, this means that if $\operatorname{Co}(\mathcal{A}, \mathcal{E})$ is the category with objects the covers $\kappa: \mathcal{A} \mapsto \mathcal{E}$ and natural morphisms, then $\pi: \mathcal{A} \mapsto \mathcal{E}$ is an initial object. (In fact, it easily follows that each object is a zero object.)

Once both theorems are established, we obtain the affirmative answer of the question of Cardinali and Sastry as a corollary of Theorem 1.1.

After that, we proceed our study of covers. We first construct an abstract semipartial geometry from a $\theta$-cover, which generalizes a construction of Brown [1]. From a number of general results, we obtain a second proof of Theorem 1.2, and we develop a second approach to answer the question of Cardinali and Sastry.

In a last section, we show that the Kantor-Knuth generalized quadrangles contain subquadrangles that are 1-subtended, an unknown result. This in its turn implies 
that no new semipartial geometries can arise from Brown's construction in the case of the Kantor-Knuth quadrangles.

In an appendix we will consider a related problem, namely the extension problem of automorphisms of subquadrangles.

\section{DEFINITIONS}

2.1. Quadrangles. A generalized quadrangle (GQ) of order $(s, t)$, where $s$ and $t$ are cardinal numbers, is a point-line incidence geometry with the following properties:

(a) Each point is incident with $t+1$ lines and each line is incident with $s+1$ points.

(b) If $(x, L)$ is a non-incident point-line pair, there is precisely one point-line pair $(y, M)$ such that $x \mathbf{I} M \mathbf{I} y \mathbf{I} L$ (here, "I" denotes the incidence relation).

(c) Two distinct points are incident with at most one line.

If both $s$ and $t$ are at least 2, we say that the GQ is thick; otherwise it is called thin. We use the usual notation $x \sim y$ to indicate that the points $x$ and $y$ are collinear, and any point is collinear with itself. For any point $x, x^{\perp}:=\{z \mid z \sim x\}$, and for any subset $Y$ of the point set, $Y^{\perp}:=\cap_{y \in Y} y^{\perp}$; we make no distinction between $y^{\perp}$ and $\{y\}^{\perp}$. In particular, we denote $\left(Y^{\perp}\right)^{\perp}$ by $Y^{\perp \perp}$.

Finally, if $u$ and $v$ are distinct points, then $\operatorname{cl}(u, v)$ is the point set $\left\{w \mid w^{\perp} \cap\{u, v\}^{\perp \perp} \neq\right.$ $\emptyset\}$.

2.2. Subquadrangles. If $\mathcal{S}=(\mathcal{P}, \mathcal{L}, \mathbf{I})$ is a generalized quadrangle (where $\mathcal{P}$ is the point set, $\mathcal{L}$ the line set, and $\mathbf{I}$ is incidence), then a subgeometry is a triple $\Gamma=\left(\mathcal{P}^{\prime}, \mathcal{L}^{\prime}, \mathbf{I}^{\prime}\right)$ such that $\mathcal{P}^{\prime} \subseteq \mathcal{P}, \mathcal{L}^{\prime} \subseteq \mathcal{L}$, and $\mathbf{I}^{\prime}$ is the induced incidence relation.

A subquadrangle (subGQ) is a subgeometry which is a generalized quadrangle. A subquadrangle $\mathcal{S}^{\prime}$ of a generalized quadrangle $\mathcal{S}$ is full if for all its lines $U$, we have the property that any point of $\mathcal{S}$ incident with $U$ is also a point of $\mathcal{S}^{\prime}$. Dually, we speak of "ideal subquadrangles."

2.3. Ovoids. If $\mathcal{S}$ is a generalized quadrangle, an ovoid of $\mathcal{S}$ is a set of points $\mathcal{O}$ in $\mathcal{S}$ such that each line contains precisely one point of $\mathcal{O}$.

2.4. Geometrical hyperplanes. A geometrical hyperplane of a generalized quadrangle $\mathcal{S}$ is a subgeometry $\Gamma$ each line of which contains at least 2 points of $\mathcal{S}$, such that every line $L$ of $\mathcal{S}$ either contains 1 point of $\Gamma$, or all its points are points of $\Gamma$ and then $L$ is a line of $\Gamma$.

If $\mathcal{S}$ is a thick generalized quadrangle of order $(s, t)$, a geometrical hyperplane is always one of the following two:

- an ovoid (seen as a subgeometry);

- a full subquadrangle of order $(s, t / s)$, and then $t \equiv 0 \bmod s$ if both parameters are finite.

If $\Gamma$ is a geometrical hyperplane of order $(s, 1)$ of a thick generalized quadrangle of order $(s, t)$, then $t=s$. 


\section{COVERS}

3.1. Morphisms. If $\Gamma=(\mathcal{P}, \mathcal{L}, \mathbf{I})$ and $\Gamma^{\prime}=\left(\mathcal{P}^{\prime}, \mathcal{L}^{\prime}, \mathbf{I}^{\prime}\right)$ are point-line incidence geometries, then a morphism $\gamma: \Gamma \mapsto \Gamma^{\prime}$ is a map from $\mathcal{P} \cup \mathcal{L}$ to $\mathcal{P}^{\prime} \cup \mathcal{L}^{\prime}$ which sends points to points, lines to lines, and preserves incidence. If $\gamma$ is bijective and the inverse map also preserves incidence, we say $\gamma$ is an isomorphism. If $\Gamma=\Gamma^{\prime}$, an isomorphism is also called an automorphism, and the set of automorphisms of $\Gamma$ naturally forms a group under composition of maps, which is denoted by $\operatorname{Aut}(\Gamma)$.

If $\Gamma$ is a point-line geometry and $S \leq \operatorname{Aut}(\Gamma)$, then for any point $v$ of $\Gamma, S_{[v]}$ denotes the subgroup of $S$ each element of which fixes $v$ linewise. Such groups will be needed later on.

3.2. $\mathcal{A}$ and $\mathcal{E}$. Let $\mathcal{S}$ be a thick generalized quadrangle, and let $\mathcal{S}^{\prime} \hookrightarrow \mathcal{S}$ be a thick full subquadrangle of $\mathcal{S}$. Let $x$ be a point of $\mathcal{S} \backslash \mathcal{S}^{\prime}$. Then $x^{\perp} \cap \mathcal{S}^{\prime}=$ : $\mathcal{O}_{x}$ is easily seen to be an ovoid of $\mathcal{S}^{\prime}$, which we call a subtended ovoid (by $x$ ). Let $L$ be any line of $\mathcal{S} \backslash \mathcal{S}^{\prime}$ which meets $\mathcal{S}^{\prime}$ in a point $\ell$ (which is necessarily unique w.r.t. this property). Then each point of $L \backslash\{\ell\}$ subtends an ovoid in $\mathcal{S}^{\prime}$, and the set of all these ovoids is called the rosette $R_{L}$ of ovoids determined by $L$. If $\mathcal{S}^{\prime}$ is also a geometrical hyperplane, there is a natural bijection between the lines incident with $x$ and the points of $x^{\perp} \cap \mathcal{S}^{\prime}$.

Keeping the latter hypothesis, we define the geometry $\mathcal{E}$ to have as points the subtended ovoids of $\mathcal{S}^{\prime}$, and as lines the rosettes $R_{L}$. Incidence is symmetrized containment. We also define a geometry $\mathcal{A}$, which is just the affine quadrangle which arises when taking away the geometrical hyperplane $\mathcal{S}^{\prime}$ of $\mathcal{S}$.

Note that there is a natural projection

$$
\pi: \mathcal{A} \mapsto \mathcal{E}:\left\{\begin{array}{l}
x \mapsto \mathcal{O}_{x} \forall \text { points } x \\
L \mapsto R_{L} \forall \text { lines } L .
\end{array}\right.
$$

By the mere definition of $\mathcal{E}$, $\pi$ is surjective on both points and lines.

3.3. Covers. Let $\gamma: \Gamma \mapsto \Gamma^{\prime}$ be a morphism between point-line incidence geometries. Suppose both geometries are not empty - so they have either at least one point or at least one line. (In fact, since $\gamma$ is a map, it suffices to ask that $\Gamma$ is not empty.) Then $\gamma$ is a cover if $\gamma$ is locally a bijection, that is, if $x$ is any point of $\Gamma, \gamma$ induces a bijection between the lines incident with $x$, and the lines incident with $\gamma(x)$, and if $L$ is a line of $\Gamma$, it induces a bijection between the points incident with $L$ and the points incident with $\gamma(L)$. Sometimes we also say that $(\Gamma, \gamma)$ is a cover (of $\Gamma^{\prime}$ ), or even that $\Gamma$ is a cover, if the covering map is clear.

In the statement of the next lemma, by "connected point-line geometry," we mean a geometry with point set $\mathcal{P}$ and line set $\mathcal{L}$ such that any two elements in $\mathcal{P} \cup \mathcal{L}$ are contained in a path of elements in $\mathcal{P} \cup \mathcal{L}$.

Lemma 3.1. If $\Gamma^{\prime}$ is connected, then a cover $\gamma: \Gamma \mapsto \Gamma^{\prime}$ is necessarily surjective.

Proof. Let $X$ be any element of $\Gamma^{\prime}$. Since $\Gamma$ is not empty, we can take some element of $\Gamma$, say $Y$. As $\Gamma^{\prime}$ is connected, we then know there is a path in $\Gamma^{\prime}$ in which $\gamma(Y)$ and $X$ are elements. Now apply the definition of cover to conclude that $X$ is 
an image for $\gamma$.

If $\gamma: \Gamma \mapsto \Gamma^{\prime}$ is a cover, and each fiber (of lines and points) has constant size $\theta$, we say that $\gamma$, or $(\Gamma, \gamma)$, or $\Gamma$ is a $\theta$-fold cover (of $\Gamma^{\prime}$ ) or simply $\theta$-cover (of $\Gamma^{\prime}$ ). We also say that $\Gamma^{\prime}$ is $\theta$-covered by $\Gamma$.

Remark 3.2. Note that the map $\pi: \mathcal{A} \mapsto \mathcal{E}$ of the previous section is a cover. We call it the canonical cover.

\section{FACTORIZATION OF MORPHISMS, I — LOWER DECOMPOSITION}

In this section, we aim to prove the following theorem.

Theorem 4.1 (Lower decomposition). Let $S$ be a thick generalized quadrangle, and let $\mathcal{S}^{\prime} \hookrightarrow \mathcal{S}$ be a thick full subquadrangle which is a geometrical hyperplane of $\mathcal{S}$. Define $\mathcal{A}$ and $\mathcal{E}$ as above, and let $\pi: \mathcal{A} \mapsto \mathcal{E}$ be the natural projection. Then any cover $\gamma: \mathcal{A} \mapsto \mathcal{E}$ factorizes as $\gamma=\alpha \circ \pi$, with $\alpha$ an automorphism of $\mathcal{E}$.

We call this property "lower decomposition" (of $\gamma$ over $\pi$, or simply of $\gamma$ ).

Before starting with the proof, let us remark that the assumption of $\gamma$ being a cover is essential: in general, morphisms between generalized quadrangles need not be injective - e.g., map every point of a quadrangle $\Gamma$ to one and the same point $x$ of a quadrangle $\Gamma^{\prime}$, and every line of $\Gamma$ to one fixed line of $\Gamma^{\prime}$ incident with $x$. (Morphisms preserve incidence, but not necessarily non-incidence.)

We will obtain the proof of Theorem 4.1 in a series of short lemmas. First assume that $\gamma$ is as in the statement of Theorem 4.1; then $\alpha$ is uniquely determined by the expression

$$
\gamma=\alpha \circ \pi
$$

if it is well defined. Namely, if $x$ is a point or a line of $\mathcal{E}$, let $y \in \pi^{-1}(x)$; then $\alpha(x)=\gamma(y)$. If $\gamma(y)$ is independent of the choice of $y \in \pi^{-1}(x)$, then it follows easily that $\alpha$ is indeed an automorphism of $\mathcal{E}$ (see below).

Observation 4.2. If $u$ and $v$ are different points in $\mathcal{A}$ for which $u^{\perp} \cap v^{\perp}=\emptyset$ in $\mathcal{A}$, then $\pi(u)=\pi(v)$ (i.e., they subtend the same ovoids at infinity). Conversely, if $\pi(u)=\pi(v)$ for different points $u$ and $v$ in $\mathcal{A}$, then $u^{\perp} \cap v^{\perp}=\emptyset$ in $\mathcal{A}$.

Proof. Suppose that $\{u, v\}^{\perp} \cap \mathcal{A}$ is empty. As $\mathcal{S}^{\prime}$ is a geometrical hyperplane, it follows easily that, in $\mathcal{S}$, we have

$$
\{u, v\}^{\perp} \subseteq\left(u^{\perp} \cap \mathcal{S}^{\prime}\right) \cap\left(v^{\perp} \cap \mathcal{S}^{\prime}\right),
$$

hence the first statement.

Now suppose that $\pi(u)=\pi(v)$ for different points $u$ and $v$ in $\mathcal{A}$. If $v \in u^{\perp}$, then triangles arise in $\mathcal{S}$ since they subtend the same ovoid in $\mathcal{S}^{\prime}$. For, if $w \in\{u, v\}^{\perp} \cap \mathcal{A}$, then the line $v w$ of $\mathcal{S}$ contains a point $r$ of the ovoid, yielding a triangle with vertices $u, w, r$, contradiction. 
Corollary 4.3. Define the relation $\mathbf{P}$ on the points of $\mathcal{A}$ as follows:

$$
x \mathbf{P} y \text { if } x^{\perp} \cap y^{\perp} \cap \mathcal{A}=\emptyset .
$$

Then $\mathbf{P}$ is an equivalence relation.

Lemma 4.4. If $x$ is a point of $\mathcal{A}$ and $y, z$ are points in $\gamma^{-1}(\gamma(x))$, then $y^{\perp} \cap z^{\perp}=\emptyset$ in $\mathcal{A}$. (In other words, if $\gamma(y)=\gamma(z)$, then $y^{\perp} \cap z^{\perp}=\emptyset$ in $\mathcal{A}$.)

Proof. Let $\gamma(y)=\gamma(z)$, and suppose $u$ is some point of $\mathcal{A}$ such that $u \neq y \sim u \sim$ $z \neq u$. As $\gamma$ induces a local bijection between $u^{\perp}$ and $\gamma(u)^{\perp}$, it must follow that $\gamma(y u) \neq \gamma(z u)$. But then $\gamma(y) \neq \gamma(z)$, contradiction.

The next lemma proves the converse of Lemma 4.4.

Lemma 4.5. Let $u$ and $v$ be different points in $\mathcal{A}$. If $u^{\perp} \cap v^{\perp}=\emptyset$ in $\mathcal{A}$, then $\gamma(u)=$ $\gamma(v)$.

Proof. Suppose $u$ and $v$ are as in the statement, and suppose $\gamma(u) \neq \gamma(v)$. We consider the possibilities.

(1) $\gamma(u) \sim \gamma(v)$. Let $M$ be the line incident with both $\gamma(u)$ and $\gamma(v)$; as $\gamma$ is a cover, it induces a bijection between the lines incident with $u$ and the lines incident with $\gamma(u)$, so there is a unique line $U \mathbf{I} u$ such that $\gamma(U)=M$. Similarly, there is a unique line $V \mathbf{I} v$ such that $\gamma(V)=M$. As $\gamma(U)=\gamma(V)$, each point on this line has unique inverse images on both $U$ and $V$. So there is a point $w$ incident with $U$ such that $\gamma(w)=\gamma(v)$. But now $v^{\perp} \cap w^{\perp} \neq \emptyset$ (as $\mathbf{P}$ is an equivalence relation), contradicting Lemma 4.4.

(2) $\gamma(u) \nsim \gamma(v)$, and there is a point $z \in \mathcal{E}$ such that $\gamma(u) \sim z \sim \gamma(v)$. First note that such a point $z$ exists; let $\widetilde{u}$ and $\widetilde{v}$ be points in $\mathcal{A}$ such that $\pi(\widetilde{u})=\gamma(u)$ and $\pi(\widetilde{v})=\gamma(v)$. Then $\widetilde{u}^{\perp} \cap \widetilde{v} \neq \emptyset$ in $\mathcal{A}$, as otherwise $\gamma(u)=\gamma(v)$. So in $\mathcal{A}$ we can find a point $\widetilde{z}$ such that $\widetilde{u} \sim \widetilde{z} \sim \widetilde{v}$; with $z:=\pi(\widetilde{z})$, it follows that $\gamma(u) \sim z \sim \gamma(v)$. Then there are point-line pairs $\left(u^{\prime}, U\right)$ and $\left(v^{\prime}, V\right)$ such that $u \mathbf{I} U \mathbf{I} u^{\prime}$ and $v \mathbf{I} V \mathbf{I} v^{\prime}$ and $\gamma\left(u^{\prime}\right)=\gamma\left(v^{\prime}\right)=z$. It follows that $\gamma(U)=\gamma(u) z$ and $\gamma(V)=\gamma(v) z$. Some (unique) $V^{\prime} \mathbf{I} u^{\prime}$ satisfies $\gamma\left(V^{\prime}\right)=\gamma(V)$, and $V^{\prime}$ contains a (unique) point $v^{\prime \prime}$ for which $\gamma\left(v^{\prime \prime}\right)=\gamma(v)$. As $u^{\perp} \cap v^{\perp}=\emptyset$ in $\mathcal{A}, v^{\prime \prime} \neq v$, and it follows that $v^{\prime \prime} \cap v^{\perp}=\emptyset$ in $\mathcal{A}$ by Lemma 4.4. By Corollary 4.3, it then follows that $u^{\perp} \cap v^{\prime \prime}{ }^{\perp}=\emptyset$, contradiction.

By Observation 4.2 and Lemmas 4.4 and 4.5, the map $\alpha$ is well defined on points. The proof for lines becomes easier at this point.

Lemma 4.6. Let $L$ be a line of $\mathcal{E}$. Then $\gamma$ maps any line of $\pi^{-1}(L)$ to the same line of $\mathcal{E}$. Also, if $M$ is any line of $\mathcal{E}$, then $\pi$ maps any line of $\gamma^{-1}(M)$ to the same line of $\mathcal{E}$.

Proof. Let $U, V \in \pi^{-1}(L)$, and let $v \mathbf{I} V$ in $\mathcal{A}$. Then $r:=v^{\perp} \cap \mathcal{S}^{\prime}$ is a point of $\mathcal{E}$ on $L$; $\pi^{-1}(r)$ is a set of points in $\mathcal{A}$ which meets every line in $\pi^{-1}(L)$ in exactly one point of $\mathcal{A}$. All these points subtend $v^{\perp} \cap \mathcal{S}^{\prime}$. By Observation 4.2 and Lemma $4.5 \gamma$ maps all these points to $\gamma(u)$, where $u:=\pi^{-1}(r) \cap U$. It follows easily by letting $(V, v)$ vary, that all lines in $\pi^{-1}(L)$ are mapped on $\gamma(U)$. 
The proof of the second part of Lemma 4.6 is similar, but now one has to rely on Observation 4.2 and Lemma 4.4.

We are now ready to obtain the proof of Theorem 4.1.

Proof of Theorem 4.1. We have that $\alpha$ is well defined on points and lines, and it easily follows that incidence is preserved.

Remark 4.7 (The automorphism $\bar{\alpha}$ ). Note that for each line $L$ of $\varepsilon$ the lines of $\gamma^{-1}(L)$ all contain the same point $u$ of $\mathcal{S}^{\prime}$. (For, let $U, V \in \gamma^{-1}(L)$, and suppose $U \cap \mathcal{S}^{\prime} \neq V \cap \mathcal{S}^{\prime}$; then $\pi(U) \neq \pi(V)$, so that $\gamma=\alpha \circ \pi$ implies that $\gamma(U) \neq \gamma(V)$, contradiction.) If the ovoids of the rosette $L$ all share the point $u^{\prime}$, then for any rosette $M \neq L$ whose ovoids share $u^{\prime}$, the lines of $\gamma^{-1}(M)$ contain $u$. (For, suppose $U \in \gamma^{-1}(L)$ and $V^{\prime} \in \gamma^{-1}(M)$ such that $U \cap \mathcal{S}^{\prime} \neq V^{\prime} \cap \mathcal{S}^{\prime}$; then we can find a line $W$ of $\mathcal{S}$ in $\left\{U, V^{\prime}\right\}^{\perp}$ for which the points $W \cap U=: w$ and $W \cap V^{\prime}=: w^{\prime}$ are points of $\mathcal{A}$. The rosette defined by $W$ contains the points (subtended ovoids) $\mathcal{O}_{w}$ and $\mathcal{O}_{w^{\prime}}$ of $\mathcal{E}$, and so these points are collinear in $\mathcal{E}$. It follows that $\gamma(w)=\alpha\left(\mathcal{O}_{w}\right)$ and $\gamma\left(w^{\prime}\right)=\alpha\left(\mathcal{O}_{w^{\prime}}\right)$ are also collinear points in $\mathcal{E}$. Since $\gamma(w)$ is a point of the rosette $L$, and $\gamma\left(w^{\prime}\right)$ is a point of the rosette $M \neq L$, this is clearly not possible.) If $u^{\prime}=: \zeta(u)$, and we let $u$ vary, we obtain a permutation

$$
\zeta: w \mapsto w^{\prime}
$$

of the points of $\mathcal{S}^{\prime}$. For distinct collinear points $v$ and $v_{1}$ of $\mathcal{S}^{\prime}$, we have that $\zeta(v)=: v^{\prime}$ and $\zeta\left(v_{1}\right)=: v_{1}^{\prime}$ are also collinear as otherwise $v^{\prime}$ and $v_{1}^{\prime}$ belong to a common point (subtended ovoid) $\mathcal{O}^{\prime}$ of $\mathcal{E}$, and hence $\alpha^{-1}\left(\mathcal{O}^{\prime}\right)=\mathcal{O}$ contains $v$ and $v_{1}$, contradiction. Similarly, if $v^{\prime}$ and $v_{1}^{\prime}$ are distinct collinear points of $\mathcal{S}^{\prime}$, then $\zeta^{-1}\left(v^{\prime}\right)$ and $\zeta^{-1}\left(v_{1}^{\prime}\right)$ are collinear as well. It follows that the map $\zeta$ defines an automorphism $\bar{\alpha}$ of $\mathcal{S}^{\prime}$.

The next result yields more information about lower decomposition.

Corollary 4.8. Let $\operatorname{Co}(\mathcal{A}, \mathcal{E})$ be the category with objects the covers $\kappa: \mathcal{A} \mapsto \mathcal{E}$ and morphisms defined as follows:

elements of $\operatorname{hom}\left(\kappa^{\prime}: \mathcal{A} \mapsto \mathcal{E}, \kappa^{\prime \prime}: \mathcal{A} \mapsto \mathcal{E}\right)$ are morphisms $\vartheta: \mathcal{E} \mapsto \mathcal{E}$ such that the following diagram commutes:



Then $\pi: \mathcal{A} \mapsto \mathcal{E}$ is an initial object. It follows that each object is a zero object.

Proof. The fact that $\pi: \mathcal{A} \mapsto \mathcal{E}$ is initial is a direct corollary of Theorem 4.1. The fact that it is zero (so also terminal) follows from the fact that $\alpha$ (in Theorem 4.1) is an automorphism. Now consider two objects $\gamma: \mathcal{A} \mapsto \mathcal{E}$ and $\gamma^{\prime}: \mathcal{A} \mapsto \mathcal{E}$ in $\operatorname{Co}(\mathcal{A}, \mathcal{E})$. Using the fact that $\pi: \mathcal{A} \mapsto \mathcal{E}$ is a zero object, one now easily proves that there are unique $\delta: \mathcal{E} \mapsto \mathcal{E}$ and $\delta^{\prime}=\delta^{-1}: \mathcal{E} \mapsto \mathcal{E}$ such that the following diagram commutes: 


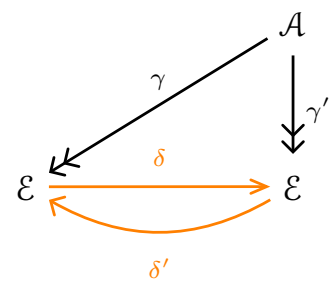

Corollary 4.9. Let $\mathcal{S}$ be a thick generalized quadrangle, and let $\mathcal{S}^{\prime} \hookrightarrow \mathcal{S}$ be a thick full subquadrangle which is a geometrical hyperplane of $\mathcal{S}$. Define $\mathcal{A}$ and $\mathcal{E}$ as above, and let $\pi: \mathcal{A} \mapsto \mathcal{E}$ be the natural projection. Then any 2-cover $\gamma: \mathcal{A} \mapsto \mathcal{E}$ factorizes as $\gamma=\alpha \circ \pi$, with $\alpha$ some automorphism of $\mathcal{E}$.

Corollary 4.10. Let $\mathcal{Q}(4, q)$ be a subquadrangle of $\mathcal{Q}(5, q)$. Define $\mathcal{A}$ and $\mathcal{E}$ as before, and let $\pi: \mathcal{A} \mapsto \mathcal{E}$ be the natural projection. Then any 2 -cover $\gamma: \mathcal{A} \mapsto \mathcal{E}$ factorizes as $\gamma=\alpha \circ \pi$, with $\alpha$ some automorphism of $\mathcal{E}$.

Proof. The proof follows from Corollary 4.9 and the fact that $\mathcal{Q}(4, q)$ is a geometrical hyperplane of $Q(5, q)$.

\section{FACTORIZATION OF MORPHISMS, II — HIGHER DECOMPOSITION}

We now handle higher decomposition.

5.1. Higher decomposition and higher extension. We keep using the notation of the previous section. In that section, we have shown that given a cover $\gamma: \mathcal{A} \mapsto \mathcal{E}$, there exists an automorphism $\alpha$ of $\mathcal{E}$ such that $\gamma=\alpha \circ \pi$, with $\pi$ the canonical cover. Now suppose that $\alpha$ itself is induced by an automorphism $\widetilde{\alpha}$ of $\mathcal{S}$ (so that $\widetilde{\alpha} \in \operatorname{Aut}(\mathcal{S})_{\mathcal{S}^{\prime}}$, and $\widetilde{\alpha}$ extends $\bar{\alpha}$, cf. Remark 4.7). It is then obvious that

$$
\gamma=\alpha \circ \pi=\pi \circ \widetilde{\alpha},
$$

and so we have (in this general setting) a "higher decomposition" of morphisms as asked (for every cover) by Cardinali and Sastry. Vice versa, if an automorphism $\widetilde{\beta}$ in $\operatorname{Aut}(\mathcal{S})_{\mathcal{S}^{\prime}}$ exists such that $\gamma=\pi \circ \widetilde{\beta}$, then $\widetilde{\beta}$ necessarily induces $\alpha$, or equivalently extends $\bar{\alpha}$, so the existence of the decomposition of Cardinali and Sastry for $\gamma$ is equivalent with the existence of an extending automorphism $\widetilde{\beta}$. In other words, we have the following result.

In the next theorem, we say that $\pi: \mathcal{A} \mapsto \mathcal{E}$ has the higher decomposition property if any cover $\gamma: \mathcal{A} \mapsto \mathcal{E}$ factorizes as $\gamma=\pi \circ \widetilde{\alpha}$ for some $\widetilde{\alpha} \in \operatorname{Aut}(\mathcal{S})$. By abuse of terminology, we also say that $\gamma: \mathcal{A} \mapsto \mathcal{E}$ has the higher decomposition property if it factorizes as above.

Theorem 5.1 (Extension property). The cover $\pi: \mathcal{A} \mapsto \mathcal{E}$ has the higher decomposition property if and only if any automorphism of $\mathcal{E}$ is induced by an automorphism of $\mathcal{S}$. 
We call the latter property the "higher extension property" (for the cover $\pi: \mathcal{A} \mapsto$ $\mathcal{E}$ ). If $\iota: \mathcal{S}^{\prime} \mapsto \mathcal{S}$ is a natural embedding of generalized quadrangles, then we say that it has the "higher extension property" if each automorphism of $\mathcal{S}^{\prime}$ extends to an automorphism of $\mathcal{S}$. Theorem 5.1 yields a criterion which makes it rather easy to check whether higher decomposition is true for all covers $\gamma: \mathcal{A} \mapsto \mathcal{E}$. We need to develop some more tools in order to apply the criterion.

Remark 5.2. It is very important to note that $\widetilde{\alpha}$ need not be unique with respect to this property: any $\widetilde{\alpha}$ which extends $\bar{\alpha}$ gives rise to the same decomposition. This is in stark contrast with the automorphism $\alpha$, which is necessarily unique. If $\widetilde{\alpha}^{\prime}$ is another such extending automorphism, $\widetilde{\alpha}\left(\widetilde{\alpha}^{\prime}\right)^{-1}$ fixes $\mathcal{S}^{\prime}$ elementwise, and it easily follows that we have a natural bijection

$$
\eta: \operatorname{Aut}(\mathcal{S})_{\left[\delta^{\prime}\right]} \mapsto E(\bar{\alpha}): \theta \longrightarrow \widetilde{\alpha} \theta
$$

where $E(\bar{\alpha})$ is the set of automorphisms of $\mathcal{S}$ that extend $\bar{\alpha}$.

Let $\operatorname{Aut}(\mathcal{S})_{\mathcal{S}^{\prime}}$ be the stabilizer of $\mathcal{S}^{\prime}$ in $\mathcal{S}$, and let $N=\operatorname{Aut}_{\left[\mathcal{S}^{\prime}\right]}$ be the subgroup of elements that fix $\mathcal{S}^{\prime}$ elementwise. Then obviously $\operatorname{Aut}(\mathcal{S})_{\mathcal{S}^{\prime}} / N \leq \operatorname{Aut}\left(\mathcal{S}^{\prime}\right)$. Each element of $\operatorname{Aut}(\mathcal{S})_{\mathcal{S}^{\prime}}$ induces an element of $\operatorname{Aut}(\mathcal{E})$ in a unique way, and modulo $N$ this happens in a faithful way. So

$$
\operatorname{Aut}(\mathcal{S})_{\mathcal{S}^{\prime}} / N \leq \operatorname{Aut}(\mathcal{E}) .
$$

The following is immediate.

Observation 5.3. If every automorphism of $\mathcal{S}^{\prime}$ extends to an automorphism of $\mathcal{S}$, then every automorphism of $\mathcal{S}^{\prime}$ induces an automorphism of $\mathcal{E}$ in a faithful manner.

Now if $\operatorname{Aut}(\mathcal{S})_{\mathcal{S}^{\prime}} / N \cong \operatorname{Aut}\left(\mathcal{S}^{\prime}\right)$ as in Observation 5.3, then the higher decomposition property is not true if and only if $\operatorname{Aut}\left(\mathcal{S}^{\prime}\right) \neq \operatorname{Aut}(\mathcal{E})$.

\section{THE ORIGINAL QUESTION}

When $\mathcal{S}=\mathcal{Q}(5, q)$ and $\mathcal{S}^{\prime}=\mathcal{Q}(4, q)$, the higher decomposition property is always true (in any characteristic), since any automorphism of $Q(4, q)$ extends in two ways (since the group of automorphisms of $2(5, q)$ fixing $Q(4, q)$ elementwise has order 2). In Brown [1, Theorem 3.3], the following is shown:

Theorem 6.1 (Brown). If $\Gamma$ is a finite thick GQ of order $\left(u, u^{2}\right)$ and $\triangle$ a subGQ of order $u$ which is 2-covered by $\Gamma \backslash \Delta$, and $\Gamma^{\prime}$ is a GQ of order $\left(u, u^{2}\right)$ with a subGQ $\Delta^{\prime}$ of order $u$ which is also 2-covered by $\Gamma^{\prime} \backslash \Delta^{\prime}$, then the geometry $\mathcal{E}$ is isomorphic to the geometry $\mathcal{E}^{\prime}$ if and only if there is an isomorphism $\kappa: \Delta \mapsto \Delta^{\prime}$ that induces an isomorphism $\kappa^{\prime}: \mathcal{E} \mapsto \mathcal{E}^{\prime}$.

As a corollary of the proof of Theorem 6.1, he deduces the following.

Corollary 6.2 (Brown). If $\Gamma$ is a finite thick GQ of order $\left(u, u^{2}\right)$ and $\Delta$ a subGQ of order $u$ which is 2-covered by $\Gamma \backslash \Delta$, then $\operatorname{Aut}(\mathcal{E})$ is the stabilizer of $\mathcal{E}$ in $\operatorname{Aut}\left(\mathcal{S}^{\prime}\right)$.

Remark 6.3. We will generalize Theorem 6.1 later on. Note that Theorem 6.1 also follows from the same reasoning as in Remark 4.7.

Combining Theorem 5.1, Observation 5.3 and Corollary 6.2 we conclude that the question of Cardinali and Sastry has an affirmative answer. 


\section{MORE ON SUBTENDED OVOIDS}

In this section, as in the sections 8-9-10-11, we only consider finite GQs.

Let $\mathcal{S}^{\prime}=\left(\mathcal{P}^{\prime}, \mathcal{B}^{\prime}, \mathbf{I}^{\prime}\right)$ be a proper subquadrangle of order $\left(s, t^{\prime}\right), s \neq 1$, of a generalized quadrangle $\mathcal{S}=(\mathcal{P}, \mathcal{B}, \mathbf{I})$ of order $(s, t)$. Then $t \geq s t^{\prime}$ and each point $x$ of $\mathcal{S}$ not in $\mathcal{S}^{\prime}$ is collinear with exactly $s t^{\prime}+1$ points of an ovoid $\mathcal{O}_{x}$ of $\mathcal{S}^{\prime}$; the ovoid $\mathcal{O}_{x}$ is said to be subtended by $x$. The points of $\mathcal{S}$ not in $\mathcal{S}^{\prime}$ are called the external points of $\mathcal{S}^{\prime}$. If $t=s t^{\prime}$, then each line of $\mathcal{S}$ is incident with a point of $\mathcal{S}^{\prime}$.

Suppose that the subtended ovoid $\mathcal{O}_{x}$ of $\mathcal{S}^{\prime}$ is subtended by $\theta$ points of $\mathcal{P} \backslash \mathcal{P}^{\prime}$; then we say that $\mathcal{O}_{x}$ is $\theta$-subtended. By $[5, \S 1.4 .1]$ we have that

$$
(\theta-1) t^{\prime} \leq s
$$

If $\theta=s+1, t^{\prime}=1$, and if $S_{x}$ is the set of the $s+1$ points subtending $\mathcal{O}_{x}$, then any point $z \in \mathcal{P} \backslash\left(\mathcal{O}_{x} \cup S_{x}\right)$ is collinear with exactly two points of $\mathcal{O}_{x} \cup S_{x}$ [5, §1.4.1(i)]. If $(\theta-1) t^{\prime}=s, t^{\prime} \neq 1$ and $\mathcal{O}_{x}=\mathcal{O}_{x^{\prime}}$ with $x \neq x^{\prime}$, then by [5, $\S 1.4 .1$ (ii)] we have that $t=s t^{\prime}$, and each point $w \notin \operatorname{cl}\left(x, x^{\prime}\right)$ is collinear with $t / s+1=t^{\prime}+1$ points of $\mathcal{O}_{x}$.

We now provide some examples of GQs $\mathcal{S}, \mathcal{S}^{\prime}$ with $\mathcal{S}^{\prime} \subset \mathcal{S}$, where each subtended ovoid $\mathcal{O}_{x}$ is $\theta$-subtended for some $\theta$.

\subsection{Examples.}

(1) Consider the subquadrangle $Q(4, q)$ of the GQ $Q(5, q)$. Then $s=t^{\prime}=q$, $t=q^{2}$, and $\theta=2$. Here $t=s t^{\prime},(\theta-1) t=s^{2}$ and $(\theta-1) t^{\prime}=s$.

(2) Consider the subquadrangle $Q(3, q)$ of the GQ $Q(5, q)$. Then $s=q, t=q^{2}$, $t^{\prime}=1$, and $\theta=q+1$. Here $t \neq s t^{\prime},(\theta-1) t \neq s^{2}$ and $(\theta-1) t^{\prime}=s$.

(3) Consider the subquadrangle $\mathcal{Q}(3, q)$ of the GQ $Q(4, q)$. Then $s=t=q$ and $t^{\prime}=1$. If $q$ is odd, then $\theta=2$. For $q$ even each subtended ovoid of $Q(3, q)$ is subtended by just one point of $Q(4, q)$.

(4) Consider the subquadrangle $\mathcal{H}\left(3, q^{2}\right)$ of the GQ $\mathcal{H}\left(4, q^{2}\right)$. Then $s=q^{2}$, $t=q^{3}, t^{\prime}=q$, and $\theta=q+1$. Here $t=s t^{\prime},(\theta-1) t=s^{2}$ and $(\theta-1) t^{\prime}=s$.

(5) It is well known that the GQ of order $\left(q, q^{2}\right)$ arising from the Kantor-Knuth flock has subquadrangles of order $q$ isomorphic to $Q(4, q)$. This also yields examples with $s=t^{\prime}=q, t=q^{2}$, and $\theta=2$; see $[1, \S 5]$.

Remark 7.1. Let $\mathcal{S}^{\prime}$ be a subquadrangle of order $s$ of the GQ $\mathcal{S}$ of order $\left(s, s^{2}\right)$, $s \neq 1$. If every subtended ovoid of $\mathcal{S}^{\prime}$ is 2 -subtended, then by [6] we have:

(i) for $s$ even we have $\mathcal{S}^{\prime} \cong 2(4, s)$, and

(ii) for $s$ odd all points of $\mathcal{S}^{\prime}$ are antiregular.

Lemma 7.2. If $t^{\prime} \neq 1, t=s t^{\prime},(\theta-1) t=s^{2}$, if every subtended ovoid of $\mathcal{S}^{\prime}$ is $\theta$ subtended, and if $x^{\prime} \in \mathcal{P} \backslash \mathcal{P}^{\prime}$ is collinear with no point of $\mathcal{O}_{x}^{\perp}$, then $\left|\mathcal{O}_{x} \cap \mathcal{O}_{x^{\prime}}\right|=t / s+1$. If $x^{\prime} \notin \mathcal{O}_{x}^{\perp}$ is collinear with a point of $\mathcal{O}_{x}^{\perp}$, then $\left|\mathcal{O}_{x} \cap \mathcal{O}_{x^{\prime}}\right|=1$.

Proof. The second part of the statement is clear. So assume that $x^{\prime} \in \mathcal{P} \backslash \mathcal{P}^{\prime}$ is collinear with no point of $\mathcal{O}_{x}^{\perp}$. Let $y \in \mathcal{O}_{x}^{\perp} \backslash\{x\}$. As $x^{\prime} \notin \operatorname{cl}(x, y),|\operatorname{cl}(x, y)|=\theta$ and $(\theta-1) t=s^{2}$, the point $x^{\prime}$ is collinear with $t / s+1$ points of $\{x, y\}^{\perp}=\mathcal{O}_{x}$, and so $\left|\mathcal{O}_{x} \cap \mathcal{O}_{x^{\prime}}\right|=t / s+1$. 


\section{The GeOMEtRy $\Gamma\left(\mathcal{S}, \mathcal{S}^{\prime}\right)=\mathcal{E}$}

Assume again that every subtended ovoid $\mathcal{O}_{x}$ of $\mathcal{S}^{\prime}$ is $\theta$-subtended, $\theta>1$. Let $L$ be a line of $\mathcal{S}$ not in $\mathcal{S}^{\prime}$ which has a point $z$ in common with $\mathcal{S}^{\prime}$. If $x_{1}, x_{2}, \ldots, x_{s}$ are the points of $L$ not in $\mathcal{S}^{\prime}$, then the ovoids $\mathcal{O}_{x_{1}}, \mathcal{O}_{x_{2}}, \ldots, \mathcal{O}_{x_{s}}$ form a rosette $R_{L}$ of ovoids in $\mathcal{S}^{\prime}$. Any two elements of $R_{L}$ have just $z$ in common and $\left|R_{L}\right|=s$. Also, the union of all ovoids of $R_{L}$ is $\mathcal{P}^{\prime}$. Now we introduce the point-line geometry $\mathcal{E}=: \Gamma\left(\mathcal{S}, \mathcal{S}^{\prime}\right)=(\Omega, \Phi, \in)$, with $\Omega$ the set of all subtended ovoids of $\mathcal{S}^{\prime}$ and with $\Phi$ the set of all rosettes of subtended ovoids in $\mathcal{S}^{\prime}$.

Theorem 8.1. Let $\mathcal{S}$ be a $G Q$ of order $(s, t)$ and $\mathcal{S}^{\prime}$ a subGQ of order $\left(s, t^{\prime}\right)$. If every subtended ovoid $\mathcal{O}_{x}$ of $\mathcal{S}^{\prime}$ is $\theta$-subtended, $\theta>1$, with $t^{\prime} \neq 1, t=s t^{\prime}$, and $(\theta-1) t=s^{2}$, then the geometry $\mathcal{E}=(\Omega, \Phi, \in)$ is a semi partial geometry with parameters

$$
s^{*}=s-1, \quad t^{*}=t, \quad \alpha^{*}=\theta, \mu^{*}=\theta\left(t-t^{\prime}\right) .
$$

Proof. Every line of $\mathcal{E}$ contains $s$ points of $\mathcal{E}$, every point of $\mathcal{E}$ is contained in $s t^{\prime}+1=t+1$ lines of $\mathcal{E}$, and from Lemma 7.2 it easily follows that any two distinct points of $\mathcal{E}$ are incident with at most one line of $\mathcal{E}$.

Ler $R_{L} \in \Phi, \mathcal{O}_{x} \in \Omega$ and $\mathcal{O}_{x} \notin R_{L}$. Let $z$ be the common point of the ovoids in $R_{L}$, and let $x=x_{1}, x_{2}, \ldots, x_{\theta}$ be the elements of $\mathcal{S}$ subtending $\mathcal{O}_{x}$. If $x$ and $z$ are collinear in $\mathcal{S}$, then also $x_{i}$ and $z$, with $i=1,2, \ldots, \theta$, are collinear in $\mathcal{S}$. In such a case the point $\mathcal{O}_{x}$ of $\mathcal{E}$ is collinear with no point of the line $R_{L}$ of $\mathcal{E}$. Now assume that $x$ and $z$ are not collinear in $\mathcal{S}$; then $x_{i}$ and $z$, with $i=1,2, \ldots, \theta$, are not collinear in $\mathcal{S}$. Let $u_{i} \sim x_{i}$, with $u_{i} \mathbf{I} L, i=1,2, \ldots, \theta$. As $\left\{x_{i}, x_{j}\right\}^{\perp}=\mathcal{O}_{x}, i \neq j$, we have $u_{i} \neq u_{j}$. If $M_{i}=x_{i} u_{i}$, with $i=1,2, \ldots, \theta$, then the rosettes $R_{M_{i}}$ are the lines of $\mathcal{E}$ which contain $\mathcal{O}_{x}$ and are concurrent with $R_{L}$ in $\mathcal{E}$. Remark that these $\theta$ rosettes are independent of the choice of the line $L^{\prime}$ with $R_{L}=R_{L^{\prime}}$. Consequently, $\alpha^{*}=\theta$.

Now let $\mathcal{O}_{x}$ and $\mathcal{O}_{x^{\prime}}$ be two points of $\mathcal{E}$ which do not belong to a common rosette of $\Phi$. Let $x=x_{1}, x_{2}, \ldots, x_{\theta}$ be the elements of $\mathcal{S}$ subtending $\mathcal{O}_{x}$ and let $x^{\prime}=$ $x_{1}^{\prime}, x_{2}^{\prime}, \ldots, x_{\theta}^{\prime}$ be the elements of $\mathcal{S}$ subtending $\mathcal{O}_{x^{\prime}}$. The points of $\left\{x_{i}, x_{j}^{\prime}\right\}^{\perp}$ in $\mathcal{S}$ but not in $\mathcal{S}^{\prime}$, with $i, j=1,2, \ldots, \theta$, subtend the ovoids collinear with $\mathcal{O}_{x}$ and $\mathcal{O}_{x^{\prime}}$ in $\mathcal{E}$. Clearly, we may choose $x_{i}$, say $x_{i}=x$. By Lemma 7.2 we have $\left|\mathcal{O}_{x} \cap \mathcal{O}_{x_{j}^{\prime}}\right|=$ $\left|\mathcal{O}_{x} \cap \mathcal{O}_{x^{\prime}}\right|=t / s+1=t^{\prime}+1$, with $j=1,2, \ldots, \theta$. Hence

$$
\left|\left(\left\{x, x_{1}^{\prime}\right\}^{\perp} \cup \ldots \cup\left\{x, x_{\theta}^{\prime}\right\}^{\perp}\right) \backslash \mathcal{P}^{\prime}\right|=\theta\left(t-t^{\prime}\right) .
$$

We conclude that $\mu^{*}=\theta\left(t-t^{\prime}\right)$, and this proves the theorem.

\subsection{Examples.}

(1) Considering the items (1) and (5) of $\S \S 7.1$, we obtain semi partial geometries with parameters

$$
s^{*}=q-1, \quad t^{*}=q^{2}, \quad \alpha^{*}=2, \quad \mu^{*}=2 q(q-1) .
$$

(2) Considering the item (4) of $\S \S 7.1$, we obtain a semi partial geometry with parameters

$$
s^{*}=q^{2}-1, \quad t^{*}=q^{3}, \quad \alpha^{*}=q+1, \quad \mu^{*}=q(q+1)\left(q^{2}-1\right) .
$$


Remark 8.2. For the geometries described in the previous examples, we also refer to Brown [1] and Hirschfeld and Thas [4].

\section{UNIQUENESS OF COVERS}

Consider again the geometry $\mathcal{E}=\Gamma\left(\mathcal{S}, \mathcal{S}^{\prime}\right)=(\Omega, \Phi, \in)$. Then $\mathcal{E}$ is $\theta$-covered by the geometry $\mathcal{A}=\mathcal{S} \backslash \mathcal{S}^{\prime}=\left(\mathcal{P} \backslash \mathcal{P}^{\prime}, \mathcal{B} \backslash \mathcal{B}^{\prime}, \mathbf{I}^{\prime \prime}\right)$, with $\mathbf{I}^{\prime \prime}$ the restriction of $\mathbf{I}$ to $\left(\left(\mathcal{P} \backslash \mathcal{P}^{\prime}\right) \times\left(\mathcal{B} \backslash \mathcal{B}^{\prime}\right)\right) \cup\left(\left(\mathcal{B} \backslash \mathcal{B}^{\prime}\right) \times\left(\mathcal{P} \backslash \mathcal{P}^{\prime}\right)\right)$; the geometry $\mathcal{A}$ through the mapping $\gamma: \mathcal{A} \mapsto \mathcal{E}$ is a $\theta$-cover of $\mathcal{E}$.

The following theorem generalizes Theorem 3.3 of [1].

Theorem 9.1. If $\gamma: \mathcal{C} \mapsto \mathcal{E}$ is any $\theta$-cover of $\mathcal{E}$ without triangles, then there exists a GQ $\chi$, a subquadrangle $\chi^{\prime}$ of $\chi$ and an isomorphism $\sigma^{*}$ from $\chi^{\prime}$ to $\mathcal{S}^{\prime}$, such that $\chi \backslash \chi^{\prime}=\mathcal{C}$ and $\Gamma\left(\chi, \chi^{\prime}\right)^{\sigma^{*}}=\mathcal{E}$.

Proof. It is clear that each line of $\mathcal{C}$ has $s$ points and that each point of $\mathcal{C}$ is on $t+1$ lines.

Let $x$ be a point of $\mathcal{S}^{\prime}$. The lines of $\mathcal{S}$ not in $\mathcal{S}^{\prime}$ containing $x$ define $\left(t-t^{\prime}\right) / \theta$ rosettes by canonical projection, and the set of these rosettes is covered by a set of $t-t^{\prime}$ mutually disjoint lines of $\mathcal{C}$. The set of these $t-t^{\prime}$ lines of $\mathcal{C}$ is by definition a point $x^{*}$ of $\chi^{\prime}$. If we consider $s+1$ collinear points $x_{1}, x_{2}, \ldots, x_{s+1}$ of $\mathcal{S}^{\prime}$, then the corresponding points $x_{1}^{*}, x_{2}^{*}, \ldots, x_{s+1}^{*}$ constitute a line $M^{*}$ of $\chi^{\prime}$. In such a case the lines of the corresponding $s+1$ sets of $t-t^{\prime}$ lines of $\mathcal{C}$ are mutually disjoint. Each point of $\chi^{\prime}$ is contained in $t^{\prime}+1$ lines of $\chi^{\prime}$. The incidence structure $\chi^{\prime}$ is isomorphic to the GQ $\mathcal{S}^{\prime}$, and

$$
\sigma^{*}: x^{*} \mapsto x
$$

defines an isomorphism from $\chi^{\prime}$ to $\mathcal{S}^{\prime}$.

Let us now extend the geometry $\mathcal{C}$ to a geometry $\chi$. The line set of $\chi$ is the union of the line set of $\mathcal{C}$ and the line set of $\chi^{\prime}$; the point set of $\chi$ is the union of the point set of $\mathcal{C}$ and the point set of $\chi^{\prime}$. A line $N$ of $\mathcal{C}$ is incident with a point $x^{*}$ of $\chi^{\prime}$ if $N$ belongs to the set $x^{*}$; the other incidences are the incidences of $\mathcal{C}$ and $\chi^{\prime}$. Each line of $\chi$ contains $s+1$ points of $\chi$, and each point of $\chi$ is contained in $t+1$ lines of $\chi$. The geometry $\chi$ contains $(s+1)(s t+1)$ points and $(t+1)(s t+1)$ lines.

Assume, by way of contradiction, that the non-collinear points $z_{1}, z_{2}, z_{3}$ of $\chi$ form a triangle. As we assumed that $\mathcal{C}$ does not contain triangles, at least one of the points $z_{1}, z_{2}, z_{3}$ belongs to $\chi^{\prime}$; also at least one of the points $z_{1}, z_{2}, z_{3}$ belongs to $\mathcal{C}$ (as $\chi^{\prime}$ has no triangles as well). Let $z_{1}, z_{2}$ belong to $\mathcal{C}$, and $z_{3}$ to $\chi^{\prime}$. The points $z_{1}, z_{2}$ cover ovoids $\mathcal{O}_{u_{1}}, \mathcal{O}_{u_{2}}$ of $\mathcal{S}^{\prime}$ with $\mathcal{O}_{u_{1}} \cap \mathcal{O}_{u_{2}}=\{u\}$, and with $z_{3}$ corresponds a point $\widetilde{z_{3}}$ of $\mathcal{S}^{\prime}$ which belongs to $\mathcal{O}_{u_{1}}$ and $\mathcal{O}_{u_{2}}$. So $u=\widetilde{z_{3}}$ and the line $z_{1} z_{2}$ of $\chi$ contains the point $z_{3}$ of $\chi$, a contradiction. Next, let $z_{1}, z_{2}$ belong to $\chi^{\prime}$, and $z_{3}$ to $\mathcal{C}$. With $z_{3}$ corresponds an ovoid $\mathcal{O}_{u_{3}}$ of $\mathcal{S}^{\prime}$, and with the lines $z_{1} z_{3}$ and $z_{2} z_{3}$ correspond rosettes containing $\mathcal{O}_{u_{3}}$. Hence with $z_{1}, z_{2}$ correspond points $\widetilde{z_{1}}, \widetilde{z_{2}}$ of $\mathcal{O}_{u_{3}}$. As $z_{1} \sim z_{2}$ in $\chi^{\prime}$, also $\widetilde{z_{1}} \sim \widetilde{z_{2}}$ in $\mathcal{S}^{\prime}$, a contradiction as $\widetilde{z_{1}}$ and $\widetilde{z_{2}}$ are points of an ovoid. Consequently $\chi$ does not contain triangles. 
From the foregoing it follows that $\chi$ is a GQ of order $(s, t)$ and that $\Gamma\left(\chi, \chi^{\prime}\right)^{\sigma^{*}}=\mathcal{E}$.

\section{PARTiCUlar CASE}

Assume that $\gamma: \mathcal{A} \mapsto \mathcal{E}$ is a $\theta$-cover of $\mathcal{E}$ (which is not necessarily the canonical projection). Let $x$ be a point of $\mathcal{S}^{\prime}$. The lines of $\mathcal{S}$ not in $\mathcal{S}^{\prime}$ containing $x$ define $\left(t-t^{\prime}\right) / \theta$ rosettes by canonical projection, and these rosettes are covered by $t-t^{\prime}$ mutually disjoint lines of $\mathcal{A}$. The set of these $t-t^{\prime}$ lines is a point $x^{*}$ of $\chi^{\prime}$.

Lemma 10.1. The $t-t^{\prime}$ lines of $\mathcal{A}$ in $x^{*}$ are incident (in $\mathcal{S}$ ) with a common point $x^{* *}$ of $\mathcal{S}^{\prime}$.

Proof. Assume, by way of contradiction, that the distinct collinear points $x, y$ of $\mathcal{A}$ define a common ovoid of $\mathcal{S}^{\prime}$ (through $\gamma$ ). This contradicts the fact that the $s$ points of the line $x y$ of $\mathcal{A}$ define the $s$ distinct ovoids of a rosette in $\mathcal{S}^{\prime}$.

Let $L, M$ be distinct lines of $\mathcal{A}$ contained in $x^{*}$ and assume, by way of contradiction, that $L$ and $M$, considered as lines of $\mathcal{S}$, are incident with distinct points $l$ and $m$ of $\mathcal{S}^{\prime}$. Let $y$ be a point of $L, z$ a point of $M$, with $y \sim z$. The ovoids of $\mathcal{S}^{\prime}$ defined by $y$ and $z$ belong to a common rosette $R$. Let $R(L)$ be the rosette defined by $L$ and let $R(M)$ be the rosette defined by $M$.

First, assume that $R(L)=R(M)$. The ovoids defined by $y$ and $z$ belong to $R(L)$, $R(M)$ and $R$. Hence $R=R(L)$, a contradiction as $L$ and $y z$ are not disjoint in $\mathcal{A}$.

Next, assume that $R(L) \neq R(M)$. Let $O(y)$ be the ovoid defined by $y$ and let $O(z)$ be the ovoid defined by $z$. If $y^{\prime}$ is any point subtending $O(y)$, then $y^{\prime}$ is collinear in $\mathcal{S}$ with at least one point $z^{\prime}$ subtending $O(z)$. So in $\mathcal{S}$ there arises a triangle with vertices $y^{\prime}, z^{\prime}$ and $O(y) \cap O(z)$, a contradiction.

We conclude that the $t-t^{\prime}$ lines of $\mathcal{A}$ in $x^{*}$ are incident with a common point $x^{* *}$ of $\mathcal{S}^{\prime}$.

Theorem 10.2. The generalized quadrangles $\chi^{\prime}$ and $\mathcal{S}^{\prime}$ can be identified.

Proof. We may identify the point $x^{*}$ of $\chi^{\prime}$ with the point $x^{* *}$ of $\mathcal{S}^{\prime}$. Let $x^{*}$ and $y^{*}$ be distinct noncollinear points of $\chi^{\prime}$. Assume by way of contradiction, that $x^{* *}$ and $y^{* *}$ are collinear in $\mathcal{S}^{\prime}$. Then the points $x=\left(x^{*}\right)^{\sigma^{*}}$ and $y=\left(y^{*}\right)^{\sigma^{*}}$ belong to some ovoid $\mathcal{O}_{z}$ of $\mathcal{S}^{\prime}$. If $z^{\prime}$ is a point of $\mathcal{C}$ defining $\mathcal{O}_{z}$, then $z^{\prime} \sim x^{* *}$ and $z^{\prime} \sim y^{* *}$ in $\mathcal{S}$. hence there arises a triangle in $\mathcal{S}$, a contradiction.

Note that $x^{* *} \mapsto x$ is the automorphism $\bar{\alpha}$ of $\mathcal{S}^{\prime}$; see Remark 4.7. For the permutation $x^{* *} \mapsto x$ also the notation $\sigma^{*}$ will be used. Hence Theorem 10.2 leads to another proof of the lower decomposition property.

We keep using the same notation for the rest of this section. 
Condition (C). Consider again the geometry $\mathcal{E}=\Gamma\left(\mathcal{S}, \mathcal{S}^{\prime}\right)=(\Omega, \Phi, \in)$. Let $M_{1}, M_{2}$, $\ldots, M_{r}$, with $1 \leq r \leq \theta$, be lines of $\mathcal{S}$ covering a common rosette in $\mathcal{S}^{\prime}$ and let $L$ be a line of $\mathcal{S}$ not in $\mathcal{S}^{\prime}$, which is not concurrent with $M_{1}, M_{2}, \ldots, M_{r}$ in $\mathcal{S}$. Put $\mathcal{M}:=\left\{M_{1}, M_{2}, \ldots, M_{r}\right\}$. Assume that $x_{0}$ is the common point of $M_{i}$ and $\mathcal{S}^{\prime}$, with $i=1,2, \ldots, r$, and that $x_{1}, x_{2}, \ldots, x_{\alpha}$ are $\alpha$ points of $\mathcal{S} \backslash \mathcal{S}^{\prime}$ on the lines $M_{1}, M_{2}, \ldots, M_{r}$, with $\alpha \in\{s-1, s\}$, and where for each $i$ the line $M_{i}$ contains at least one of the points $x_{1}, x_{2}, \ldots, x_{\alpha}$. Let $N_{i}$ be the line incident with $x_{i}$ and concurrent with $L$, with $i=0,1, \ldots, \alpha$. Assume that all points $N_{i} \cap L$ are distinct, so that there arise $s$ or $s+1$ points on $L$. The set consisting of all points of $\mathcal{S}^{\prime}$ incident with the lines $N_{0}, N_{1}, \ldots, N_{\alpha}$ is denoted by $\mathcal{W}_{L, \mathcal{M}}$ if $N_{0}$ is not a line of $\mathcal{S}^{\prime}$ and by $\overline{\mathcal{W}_{L, \mathcal{M}}}$ if $N_{0}$ is a line of $\mathcal{S}^{\prime}$. We say that Condition (C) is satisfied if no sets of type $\mathcal{W}_{L^{\prime}, \mathcal{M}^{\prime}}$ with $\left|\mathcal{M}^{\prime}\right|=1$ and of type $\mathcal{W}_{L, \mathcal{M}}$ with $|\mathcal{M}|>1$, are isomorphic in $\mathcal{S}^{\prime}$, and if additionally no sets of type $\overline{\mathcal{W}_{L^{\prime}, \mathcal{M}^{\prime}}}$ with $\left|\mathcal{M}^{\prime}\right|=1$ and of type $\overline{\mathcal{W}_{L, \mathcal{M}}}$ with $|\mathcal{M}|>1$, are isomorphic in $\mathcal{S}^{\prime}$.

The next theorem shows that if $(C)$ holds, higher decomposition is possible.

Theorem 10.3. Consider a $\theta$-cover $\gamma: \mathcal{A} \mapsto \mathcal{E}$ and suppose that Condition (C) is satisfied. Then there exists an automorphism $\sigma$ of $\mathcal{S}$ with $\sigma^{*}$ the restriction of $\sigma$ to $\mathcal{S}^{\prime}$.

Proof. We will construct an automorphism $\sigma$ of $\mathcal{S}$, such that $\sigma^{*}$ is the restriction of $\sigma$ to $\mathcal{S}^{\prime}$.

Choose a point $z$ of $\mathcal{A}$ and choose a line $N$ of $\mathcal{S}$ incident with $z$; the point of $\mathcal{S}^{\prime}$ incident with $N$ is denoted by $x^{*}$. The point $z$ covers an ovoid $\mathcal{O}(z)$ of $\mathcal{S}^{\prime}$ and $\mathcal{O}(z)$ is subtended by the points $z^{\prime}=z_{1}, z_{2}, \ldots, z_{\theta}$ of $\mathcal{S}$. Let $z^{\sigma}=z^{\prime}$. Then the line $N^{\sigma}$ is the line $N^{\prime}=z^{\prime} x$, with $x=x^{* \sigma}=x^{* \sigma^{*}}$. For any line $T$ of $\mathcal{S}^{\prime}$, we define $T^{\sigma}=T^{\sigma^{*}}$.

Now let $y$ be a point of $z x^{*}$, with $x^{*} \neq y \neq z$. The point $y$ covers an ovoid $\mathcal{O}(y)$ of $\mathcal{S}^{\prime}$ and $\mathcal{O}(y)$ is subtended by a unique point $y^{\prime}$ of $N^{\prime}$; let $y^{\prime}=y^{\sigma}$. Next, consider a point $v$ of $\mathcal{A}$, which is not collinear with $x^{*}$. Let $V$ be the line incident with $v$ and concurrent with $N$, let $w^{*}$ be the common point of $V$ and $\mathcal{S}^{\prime}$, and let $a$ be the common point of $N$ and $V$. The point $v$ covers and ovoid $\mathcal{O}(v)$ of $\mathcal{S}^{\prime}$ and $\mathcal{O}(v)$ is subtended by a point $v^{\prime}$ of the line $V^{\prime}=w a^{\sigma}$ (note that this is indeed a line), with $w^{* \sigma}=w$. Let $V^{\prime}=V^{\sigma}$ and $v^{\prime}=v^{\sigma}$. Then $\sigma$ is defined for all points of $\mathcal{S}$ not colllinear with $x^{*}$, for all points of the line $N$, for all points of $\mathcal{S}^{\prime}$, for the line $N$, for all lines concurrent with $N$ but not containing $x^{*}$, and for all lines of $\mathcal{S}^{\prime}$; for all these points and lines $\sigma$ preserves incidence.

Next, let $U$ be a line of $\mathcal{S}$ not concurrent with $N$ and not contained in $\mathcal{S}^{\prime}$. Let $u^{*}$ be the common point of $U$ and $\mathcal{S}^{\prime}$. The line $U$ of $\mathcal{A}$ covers a rosette consisting of $s$ ovoids $\mathcal{O}\left(u_{1}\right), \mathcal{O}\left(u_{2}\right), \ldots, \mathcal{O}\left(u_{s}\right)$ of $\mathcal{S}^{\prime}$, with $u_{1}, u_{2}, \ldots, u_{s}$ the points of $\mathcal{A}$ incident with $U$. These ovoids have as common point $u=u^{* \sigma}$. The ovoid $\mathcal{O}\left(u_{i}\right)$ is subtended by the points $u_{i}^{1}, u_{i}^{2}, \ldots, u_{i}^{\theta}$ of the lines $U_{1}, U_{2}, \ldots, U_{\theta}$ incident with $u$, $i=1,2, \ldots, s$. The points $u_{i}$ which are not collinear with $x^{*}$ are mapped by $\sigma$ onto points $u_{i}^{\sigma}$, where $u_{i}^{\sigma}$ is incident with a line $U_{1}, U_{2}, \ldots, U_{\theta}$. Call the subset of $\left\{U_{1}, U_{2}, \ldots, U_{\theta}\right\}$ which consists of the lines containing the points $u_{i}^{\sigma}$, with $u_{i} \not x^{*}$ and $i \in\{1,2, \ldots, s\}, \mathcal{U}^{\prime}$, and put $r:=\left|\mathcal{U}^{\prime}\right|$. The lines concurrent with $N$ and $U$ which are not incident with $x^{*}$ are mapped by $\sigma$ onto the lines incident with $u_{i}^{\sigma}$, where $u_{i} \nsim x^{*}$, and concurrent with $N^{\sigma}, i \in\{1,2, \ldots, s\}$, and the line incident with $u^{* \sigma}$ and concurrent with $N^{\sigma}$. So there arise either $s$ or $s+1$ lines intersecting 
$N^{\sigma}$ in distinct points. These two sets of lines define isomorphic point sets $\mathcal{W}_{N,\{U\}}$ and $\mathcal{W}_{N^{\sigma}, u^{\prime}}$, or $\overline{\mathcal{W}_{N,\{U\}}}$ and $\overline{\mathcal{W}_{N^{\sigma}, u^{\prime}}}$ in $\mathcal{S}^{\prime}$. As Condition (C) is satisfied we have $r=1$. So the points $u_{i}^{\sigma}$, with $u_{i} \nsim x^{*}$, are incident with the line $U_{j}$ for some $j \in\{1,2, \ldots, \theta\}$, say $U_{j}=U_{1}$. Now we put $U^{\sigma}=U_{1}$.

We conclude that $\sigma$ is defined on all lines of $\mathcal{S}$ not incident with $x^{*}$ and on all points not collinear with $x^{*}$. For all these points and lines $\sigma$ preserves incidence. One easily shows that such a $\sigma$ can be extended to an automorphism of $\mathcal{S}$ for which $\sigma^{*}$ is the restriction to $\mathcal{S}^{\prime}$.

Remark 10.4. Note that the proof implies that there are $\theta$ such automorphisms $\sigma$.

The first part of the next theorem yields the higher decomposition property for the case $\mathcal{S} \cong 2(5, q), \mathcal{S}^{\prime} \cong 2(4, q)$.

Theorem 10.5. (i) If $\mathcal{S}=\mathcal{Q}(5, q), \mathcal{S}^{\prime}=\mathcal{Q}(4, q), \mathcal{C}=\mathcal{A}=\mathcal{S} \backslash \mathcal{S}^{\prime}$, then there exists an automorphism $\sigma$ of $\mathcal{Q}(5, q)$ with $\sigma^{*}$ the restriction of $\sigma$ to $Q(4, q)$.

(ii) If $\mathcal{S}=\mathcal{H}\left(4, q^{2}\right), \mathcal{S}^{\prime}=\mathcal{H}\left(3, q^{2}\right), \mathcal{C}=\mathcal{A}=\mathcal{S} \backslash \mathcal{S}^{\prime}$, then there exists an automorphism $\sigma$ of $\mathcal{H}\left(4, q^{2}\right)$ with $\sigma^{*}$ the restriction of $\sigma$ to $\mathcal{H}\left(3, q^{2}\right)$.

Proof. By Theorem 10.3 it is sufficient to show that Condition (C) is satisfied. In both cases sets $\mathcal{W}_{L, \mathcal{M}}$ and $\overline{\mathcal{W}_{L, \mathcal{M}}}$ are contained in a plane of the ambient projective space of $\mathcal{S}^{\prime}$, while sets $\mathcal{W}_{L, \mathcal{M}}$ and $\overline{W_{L, \mathcal{M}}}$, with $|\mathcal{M}|>1$, are never contained in a plane of the ambient space of $\mathcal{S}^{\prime}$. It follows that Condition (C) is satisfied, and so the theorem is proved.

\section{SUbTENDING IN THE KANTOR-KNUTH QUADRANGLES}

Let $\mathcal{S}$ be a nonclassical Kantor-Knuth GQ of order $\left(q, q^{2}\right)$. By [10], Aut $(\mathcal{S})$ does not act transitively on the subquadrangles of order $q$ (which are all isomorphic to $\mathcal{Q}(4, q)$ ). Let $\Gamma_{1}$ and $\Gamma_{2}$ be two such subGQs in different Aut(S)-orbits (it is known that there are two such orbits). If they are both doubly subtended, then we obtain two SPGs $\varepsilon_{1}$ and $\varepsilon_{2}$. Suppose they are isomorphic. Then if there would be an element $\kappa$ in $\operatorname{Aut}(\mathcal{S})$ sending $\mathcal{E}_{1}$ to $\mathcal{E}_{2}$, and so $\Gamma_{1}$ to $\Gamma_{2}$, we have a contradiction. So under this assumption (which we call (\#) for now), the SPGs are not isomorphic, and in [1] Brown only found one particular subGQ which was doubly subtended. From this point of view it is of fundamental interest to check whether all $2(4, q)$ subGQs of $\mathcal{S}$ are doubly subtended.

In this section we will show that the elements of one of these orbits do not have this property, so that no new SPGs can arise.

Below, $\mathcal{S}$ is a nonclassical Kantor-Knuth GQ of order $\left(q, q^{2}\right)-$ see [7, $\S 4.5$ and $\S 4.7 .3]$. It has a line [ $\infty]$ which is fixed by $\operatorname{Aut}(\mathcal{S})$, and there are $q^{3}+q^{2}$ subGQs of order $q$, all isomorphic to $2(4, q)$, that contain the line [ $\infty]$. There are no other subquadrangles of order $q$. All this can be found in [7, §5.1].

From [7, Theorem 5.2.1(b)] the following result on orbits of subquadrangles can be deduced. 
Orbit property There are two Aut $(\mathcal{S})$-orbits of subquadrangles of order $q$; one of size $2 q^{2}$ (called $\Omega_{1}$ ), one of size $(q-1) q^{2}$ (called $\Omega_{2}$ ). In particular, if $\Gamma$ is a line grid with parameters $(q, 1)$ and containing $[\infty]$, then there are $2 \Omega_{1}$ subquadrangles containing $\Gamma$, and $q-1 \Omega_{2}$-subquadrangles containing $\Gamma$.

In Brown [1], it is shown that the elements in $\Omega_{1}$ are all doubly subtended. Now suppose that the elements in $\Omega_{2}$ also are. Consider any grid $\Gamma$ as above. As we assume that all $q+1$ subquadrangles of order $q$ containing $\Gamma$ are doubly subtended, we obtain precisely $q+1$ distinct involutory automorphisms of $\mathcal{S}$, each fixing one of the subquadrangles of order $q$ containing $\Gamma$ pointwise. So the group $\vartheta$ generated by these involutions has at least size $q+2$. Each element of $\vartheta$ fixes $\Gamma$ elementwise. Now $\vartheta$ acts on the subquadrangles $S_{1}$ and $S_{2}$ in $\Omega_{1}$ that contain $\Gamma$. In particular, it follows that if $\theta_{1}$ is the involution that fixes $\delta_{1}$ elementwise, we have

$$
2 \geq\left|\vartheta_{\mathcal{S}_{1}} /\left\langle\theta_{1}\right\rangle\right| \geq \frac{q+2}{4},
$$

since the subgroup of $Q(4, q)$ ( $q$ odd) that fixes a grid with parameters $(q, 1)$ elementwise, has size 2 .

It follows that $q \leq 6$, so that $q$ is a prime. In those cases, $\mathcal{S} \cong \mathcal{Q}(5, q)[7, \S 4.5]$, contradiction.

We have essentially proven the following.

Theorem 11.1. No elements of $\Omega_{2}$ are doubly subtended. In particular, each element of $\Omega_{2}$ contains $(q+1) q^{2}(q-1)$ Kantor-Knuth ovoids which are 1-subtended.

Proof. The proof follows from the discussion preceding the statement of the theorem, plus the fact that for any subquadrangle of order $q$ containing [ $\infty]$, any subtended ovoid is a Kantor-Knuth ovoid by [8, Theorem 14].

In a forthcoming paper, we will study the assumption (\#), the Kantor-Knuth quadrangles and the associated SPGs in more depth. 


\section{APPENDIX A. EXTENSION IN NONCLASSICAL CASES}

In this appendix, we digress to focus on the extension property for $\mathcal{S}^{\prime}$ instead of $\mathcal{E}$.

A.1. TGQs. A translation generalized quadrangle (TGQ) is a generalized quadrangle $\delta$ which has a point $u$ called translation point such that there is an abelian automorphism group $A \leq \operatorname{Aut}(\mathcal{S})_{[u]}$ that acts sharply transitively on the points not collinear with $u$. For details about the results and notions on TGQs used in this section, we refer to the monograph [7].

Let $\mathcal{S}$ be any thick TGQ of order $\left(q, q^{2}\right)$ which is not classical, and which has a subGQ $\mathcal{S}^{\prime}$ isomorphic to $\mathcal{Q}(4, q)$ (if a GQ of order $\left(q, q^{2}\right)$ has a classical subGQ of order $\left(s^{\prime}, t^{\prime}\right)$ which is a geometrical hyperplane, then $s^{\prime}=t^{\prime}=q$, and the subGQ must be isomorphic to $Q(4, q)$ ). It is well known (see for example [9]) that $\mathcal{S}^{\prime}$ contains all the translation points of $\mathcal{S}$ (and either there is one such point, or a line of translation points). Suppose the embedding $\iota: \mathcal{S}^{\prime} \hookrightarrow \mathcal{S}$ has the higher extension property. Then any automorphism of the $Q(4, q)$-subquadrangle extends to an automorphism of $\mathcal{S}$. In particular, it follows that $\operatorname{Aut}(\mathcal{S})_{\mathcal{S}^{\prime}}$ acts transitively on the points of $\mathcal{S}^{\prime}$, so any point of $\mathcal{S}^{\prime}$ is a translation point of $\mathcal{S}$. So $\mathcal{S}$ is classical, a contradiction.

So any such $\mathcal{S}$ gives rise to embeddings which do not have the higher extension property. Of the known examples, only the $\mathcal{Q}(5, q)$ quadrangles and the KantorKnuth quadrangles also give rise to covers which are not 1-covers (see, e.g., [10]).

A.2. Generalization to EGQs. If we leave out the term "abelian" in the definition of TGQ, we have, by definition, an elation generalized quadrangle (EGQ) with elation point $u$. Most of the standard results on EGQs can be found in the book [11].

Now consider the same situation as in the previous section, with "TGQ" replaced by "EGQ." Suppose the embedding $\iota: \mathcal{S}^{\prime} \hookrightarrow \mathcal{S}$ has the higher extension property.

Suppose first that $x$ is an elation point of $\mathcal{S}$ which is contained in $\mathcal{S}^{\prime}$. Then as above any point of $\mathcal{S}^{\prime}$ is an elation point of $\mathcal{S}$. So each point of $\mathcal{S}$ is an elation point. By the main result of [12], $\delta$ is classical, a contradiction.

Now suppose that $x$ is not contained in $\mathcal{S}^{\prime}$. Let $u \sim x$ be a point of $\mathcal{S}^{\prime}$. As $\mathcal{S}^{\prime}$ is classical, there is an automorphism $\kappa$ of $\mathcal{S}^{\prime}$ which moves $u$ to a collinear point $u^{\prime} \neq u$. Let $\widetilde{\kappa}$ be an automorphism of $\mathcal{S}$ which induces $\kappa$ in $\mathcal{S}^{\prime}$. Then $y:=x^{\widetilde{\kappa}} \neq x$. If $y \sim x, y x$ is a line of elation points of $\mathcal{S}$, so that $x y \cap \mathcal{S}^{\prime}$ is also an elation point of $\mathcal{S}$, and then we are in the previous case. If $y \not x$, each point of $\mathcal{S}$ is an elation point, and then again by [12], $\mathcal{S}$ is classical, contradiction.

A.3. Quadrangles with reguli. Let $\mathcal{S}^{\prime}$ be a GQ with finite parameters $(s, 1)$, and let it be a full geometrical hyperplane in a GQ $\mathcal{S}$. Then $\mathcal{S}$ necessarily has parameters $(s, s)$. Suppose that every automorphism of $\mathcal{S}^{\prime}$ extends to automorphisms of $\mathcal{S}$. The automorphism group of $\mathcal{S}^{\prime}$ is isomorphic to $\left(\mathbf{S}_{s+1} \times \mathbf{S}_{s+1}\right) \rtimes \mu_{2}$, where $\mathbf{S}_{\ell}$ denotes the symmetric group on $\ell$ letters, and $\mu_{2}$ is a group of order 2 . Now if $s$ is a prime power, $\mathbf{S}_{s+1} \times \mathbf{S}_{s+1}$ (by which we mean the subgroup of Aut $\left(\mathcal{S}^{\prime}\right)$ that preserves the reguli of $\left.\mathcal{S}^{\prime}\right)$ contains the natural action(s) of $\mathbf{P S L}_{2}(s) \times \mathbf{P S L}_{2}(s)$ (in the language of De Kaey and Van Maldeghem [3]), and by loc. cit., it follows that $\mathcal{S} \cong 2(4, s)$. However, in that case $\operatorname{Aut}(\mathcal{S})_{\mathcal{S}^{\prime}}$ induces $\mathbf{P} \boldsymbol{\Gamma} \mathbf{O}_{4}^{+}(s)$ (which has size $(s+1)^{2} s^{2}(s-1)^{2} \cdot 2 h$, where 
$s=p^{h}$ with $p$ prime) on $\mathcal{S}^{\prime}$. It follows that $s \in\{2,3,4\}$. For $s=2,3$, one easily sees that the higher extension property is true. However, for $s=4$, it is not: this follows immediately by comparing the sizes of $\mathbf{P} \boldsymbol{\Gamma} \mathbf{O}_{4}^{+}(4)$ and $\left(\mathbf{S}_{5} \times \mathbf{S}_{5}\right) \rtimes \mu_{2}$, but can also easily be seen by observing that the action of the latter group would imply that every ovoid of $\mathcal{S}^{\prime}$ would be subtended by at least one point of $\mathcal{S}$, and this is not possible in $Q(4,4)$.

When $s$ is a not a prime power, see the discussion below, and Remark A.1.

Suppose now that $\Delta$ is an infinite generalized quadrangle which has a full thin subquadrangle $\Omega$ of order $(\omega, 1)$ that is a geometrical hyperplane, and such that $\iota: \Omega \hookrightarrow \Delta$ has the higher extension property.

Let $\Delta$ and $\Omega$ be as above. Let $A$ be an automorphism group of $\Delta$ which induces the full automorphism group of $\Omega$ on $\Omega$. So $A$ induces $\left(\mathbf{S}\left(\Omega_{1}\right) \times \mathbf{S}\left(\Omega_{2}\right)\right) \rtimes \mu_{2}$ on $\Omega$, where $\Omega_{1}$ and $\Omega_{2}$ are the two reguli of $\Omega$, and $\mathbf{S}(S)$, with $S$ a set, denotes the symmetric group of $S$. Note that

$$
|A| \geq\left|\mathbf{S}\left(\Omega_{1}\right)\right|=\left|\mathbf{S}\left(\Omega_{2}\right)\right|>\left|\Omega_{1}\right|=\left|\Omega_{2}\right|=\omega .
$$

Now let $A_{1}$ be the subgroup of $A$ that fixes each line of $\Omega_{2}$; it induces $\mathbf{S}\left(\Omega_{1}\right)$ on $\Omega_{1}$. Let $z$ be any point of $\Delta$ which is not contained in $\Omega$. For each line $M \in \Omega_{2} \cup \Omega_{1}$, let $Z_{M}$ be the line incident with $z$ which meets $M$. Let $\mathcal{L}$ be the set of lines $\left\{Z_{L} \mid L \in\right.$ $\left.\Omega_{2}\right\}$. Then as $\Omega$ is a geometrical hyperplane, we have that $t=|\mathcal{L}|=\left|\Omega_{2}\right|=\omega$, where $(\omega, t)$ is the order of $\Delta$.

So the number of points of $\Delta$ equals $\omega$ as well. As $\left|A_{1}\right|>\omega$, we have $\left|\left(A_{1}\right)_{z}\right|>1$. But the fixed elements structure of any element $\delta \neq$ id of $\left(A_{1}\right)_{z}$ is a subquadrangle which is full and ideal, so by $[13,1.8 .2], \delta$ is the identity in $A$, contradiction. So if $\Omega$ is supposed to be a geometrical hyperplane, then no examples can arise.

Remark A.1. Note that the latter approach also works for the finite case.

The next general question naturally comes to mind, but might be hard:

Classify the infinite quadrangles $\Delta$ of order $(\omega, t)$ with $t \geq \omega$ which have a full thin subquadrangle $\Omega$ of order $(\omega, 1)$, such that $\iota: \Omega \hookrightarrow$ $\Delta$ has the higher extension property. 


\section{REFERENCES}

[1] M. R. Brown. Semipartial geometries and generalized quadrangles of order $\left(r, r^{2}\right)$, in: Finite geometry and combinatorics (Deinze, 1997), Bull. Belg. Math. Soc. Simon Stevin 5 (1998), 187-205.

[2] I. Cardinali and N. S. N. Sastry. Elliptic ovoids and their rosettes in a classical generalized quadrangle of even order, Proc. Indian Acad. Sci. Math. Sci. 126 (2016), 591-612.

[3] J. De Kaey and H. Van Maldeghem. A characterization of the split Cayley generalized hexagon $H(q)$ using one subhexagon of order (1, q), Discrete Math. 294 (2005), 109-118.

[4] J. W. P. Hirschfeld and J. A. Thas. General Galois Geometries, Springer Monographs in Mathematics.,Springer, London, 2016.

[5] S. E. Payne and J. A. Thas. Finite Generalized Quadrangles. Second edition, EMS Series of Lectures in Mathematics, European Mathematical Society (EMS), Zürich, 2009.

[6] J. A. Thas. 3-regularity in generalized quadrangles: a survey, recent results and the solution of a longstanding conjecture, Combinatorics '98 (Mondello), Rend. Circ. Mat. Palermo (2) Suppl. No. 53 (1998), 199-218.

[7] J. A. Thas, K. Thas and H. Van Maldeghem. Translation Generalized Quadrangles, Series in Pure Mathematics 26, World Scientific Publishing Co. Pte. Ltd., Hackensack, NJ, 2006.

[8] K. Thas. Translation generalized quadrangles for which the translation dual arises from a flock, Glasg. Math. J. 45 (2003), 457-474.

[9] K. Thas. Symmetry in Finite Generalized Quadrangles, Frontiers in Mathematics, Birkhäuser Verlag, Basel, 2004.

[10] K. Thas. A stabilizer lemma for translation generalized quadrangles, European J. Combin. 28 (2007), 1-16.

[11] K. Thas. A Course on Elation Quadrangles, EMS Series of Lectures in Mathematics, European Mathematical Society (EMS), Zürich, 2012.

[12] K. Thas and H. Van Maldeghem.Geometric characterizations of finite Chevalley groups of type $B_{2}$, Trans. Amer. Math. Soc. 360 (2008), 2327-2357.

[13] H. Van Maldeghem. Generalized Polygons, Monographs in Mathematics 93, Birkhäuser Verlag, Basel, 1998.

Ghent University, Department of Mathematics, KriJgslaAn 281, S22 and S25, B-9000 Ghent, BELGIUM

E-mail address: thas.joseph@gmail.com; koen.thas@gmail.com 